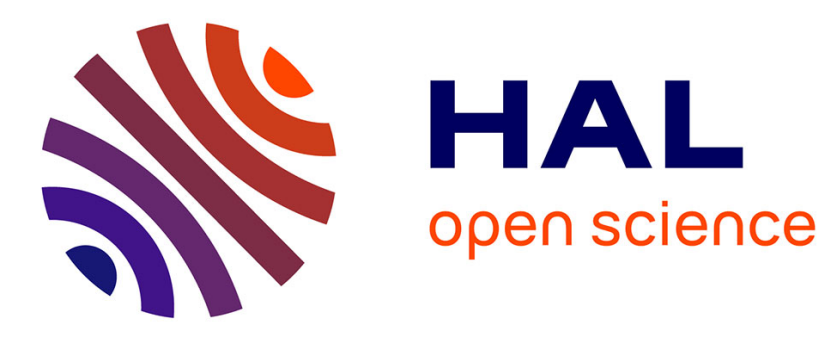

\title{
Shoulder kinetics during start-up and propulsion with a manual wheelchair within the initial phase of uninstructed training
}

Samuel Hybois, Alice Siegel, Joseph Bascou, Nicolas Eydieux, Philippe Vaslin, Hélène Pillet, Pascale Fodé, Christophe Sauret

\section{To cite this version:}

Samuel Hybois, Alice Siegel, Joseph Bascou, Nicolas Eydieux, Philippe Vaslin, et al.. Shoulder kinetics during start-up and propulsion with a manual wheelchair within the initial phase of uninstructed training. Disability and Rehabilitation: Assistive Technology, 2017, 13 (1), pp.40-46. 10.1080/17483107.2016.1278471 . hal-02185659

\section{HAL Id: hal-02185659 \\ https://hal.science/hal-02185659}

Submitted on 16 Jul 2019

HAL is a multi-disciplinary open access archive for the deposit and dissemination of scientific research documents, whether they are published or not. The documents may come from teaching and research institutions in France or abroad, or from public or private research centers.
L'archive ouverte pluridisciplinaire HAL, est destinée au dépôt et à la diffusion de documents scientifiques de niveau recherche, publiés ou non, émanant des établissements d'enseignement et de recherche français ou étrangers, des laboratoires publics ou privés. 


\section{Shoulder kinetics during start-up and propulsion with a manual wheelchair within the initial phase of uninstructed training}

Samuel Hybois, Alice Siegel, Joseph Bascou, Nicolas Eydieux, Philippe Vaslin, Hélène Pillet, Pascale Fodé \& Christophe Sauret

To cite this article: Samuel Hybois, Alice Siegel, Joseph Bascou, Nicolas Eydieux, Philippe Vaslin, Hélène Pillet, Pascale Fodé \& Christophe Sauret (2017): Shoulder kinetics during start-up and propulsion with a manual wheelchair within the initial phase of uninstructed training, Disability and Rehabilitation: Assistive Technology

To link to this article: http://dx.doi.org/10.1080/17483107.2016.1278471 


\title{
Shoulder kinetics during start-up and propulsion with a manual wheelchair within the initial phase of uninstructed training
}

\author{
Samuel Hybois ${ }^{a, b}$ (D) Alice Siegel ${ }^{a, b}$, Joseph Bascou ${ }^{a, b}$ (D), Nicolas Eydieux ${ }^{a, b}$, Philippe Vaslin ${ }^{c, d}$, Hélène Pillet ${ }^{a}$ (D), \\ Pascale Fodéb ${ }^{b}$ and Christophe Sauret ${ }^{\mathrm{a}}$ \\ ${ }^{a}$ Arts et Metiers ParisTech, Institut de Biomécanique Humaine Georges Charpak, Paris, France; ${ }^{b}$ Centre d'Etudes et de Recherche pour \\ I'Appareillage des Handicapés, Institution Nationale des Invalides, Woippy, France; 'Laboratoire d'Informatique, de Modélisation \\ et d'Optimisation des Systèmes, Clermont Université, Clermont-Ferrand, France; 'Laboratoire d'Informatique, de Modélisation et d'Optimisation \\ des Systèmes, Centre National de la Recherche Scientifique, Aubière, France
}

\begin{abstract}
Purpose: Wheelchair locomotion is constraining for the upper limbs and involves a set of motor tasks that need to be learnt by a novice user. To understand this integration process, we investigated the evolution of shoulder kinetics during start-up and propulsion within the initial phase of low-intensity uninstructed training.

Materials and methods: Seventeen novice able-bodied subjects performed a 120-min uninstructed practice distributed over 4 weeks. During the initial and final sessions, upper limbs kinematics and hand-rim kinetics were continuously collected. Inverse kinematics and dynamics coupled to a three-dimensional linked-segment model were used to compute shoulder net moments.

Results: Participants increased the speed of the wheelchair with practice. In average, an increase of shoulder net moments and mechanical work during the push phase was observed. Conversely, during the recovery phase, participants slightly increased shoulder power but maintained a similar level of shoulder loading. However, individual evolutions allowed the definition of two groups defined as: "increasers", who increased shoulder loading and mechanical work versus "decreasers", who managed to limit shoulder loading while improving the wheelchair speed.

Conclusion: These findings underline that individual adaptation strategies are essential to take into account when designing a rehabilitation protocol for wheelchair users.
\end{abstract}

> IMPLICATIONS FOR REHABILITATION

- The learning process of manual wheelchair locomotion is essential for the assimilation of motor tasks leading individuals to select their propulsion technique.

- Novice users display different learning strategies: some people increase shoulder loading very early but others spontaneously manage to increase the wheelchair speed while maintaining a constant level of shoulder loading.

- Wheelchair rehabilitation programs should be individualized to take into account the subject-specific learning strategy.
ARTICLE HISTORY

Received 21 November 2016 Accepted 30 December 2016

\section{KEYWORDS}

Biomechanics; kinetics; rehabilitation; shoulder; wheelchair

\section{Introduction}

A manual wheelchair (MWC) is a medical device that allows people with motor disabilities to recover a part of their autonomy. Nowadays, MWCs are used by more than 600,000 persons in France, representing about $1 \%$ of the French population [1]. Higher rates were reported in some other countries, such as Great-Britain where MWC users represented about $2 \%$ of the population [2]. In Brazil, some authors reported about 5 million users from a population of about 200 million people, reaching a rate of about $2.5 \%$ [3]. However, propulsion through hand-rims fixed on the rear wheels, which is the widespread propulsion mode for MWC locomotion, is a highly demanding task for the upper extremity. As a consequence, it results in a high incidence of shoulder injuries in MWC users population [4-7]. The high mechanical loads and occurrence frequency of wheelchair-related tasks throughout the day are believed to be concomitant factors related to the apparition of musculoskeletal disorders [7-11]. However, stresses on the upper extremity are not only influenced by external loads such as the forces to overcome inertial forces and motion resistances [12], but also by the propulsion technique [13-16] including both spatiotemporal parameters and joint configurations.

Hand-rim wheelchair locomotion is a set of motor tasks that needs to be learnt, often when being an adult. So to improve rehabilitation program efficiency, it is important to understand how these gross motor tasks are acquired. Numerous studies investigated the effect of various rehabilitation programs focusing on strength and physical conditioning [17-20] or on hand-rim wheelchair technique [21-23]. Several studies investigated the effect of visual [21-23] or haptic feedback [24] to reinforce the rehabilitation program efficiency with contrasted results. Others investigated the effect of uninstructed practice of hand-rim 
wheelchair propulsion [25-29] based on the assumption that subjects will naturally converge to an optimum technique minimizing energy expenditure for a given velocity. As a consequence, it may also be assumed that, at a self-selected comfortable velocity, shoulder loads would decrease throughout the learning process. This assumption is further supported by previous results where skilled hand-rim wheelchair users developed a lower propelling torque together with a higher mechanical power than unskilled subjects [30].

Generally, studies on wheelchair training program showed a decrease in oxygen consumption for a given external mechanical power, indicating an improvement in mechanical efficiency $[19,22,29,31]$. Most of the studies focused on steady-state propulsion and investigated the changes in propulsion technique associated with the improvement in mechanical efficiency. In particular, an increase in cycle duration, contact angle and a change in force direction was observed [28,29,31]. Other authors described different patterns of the hand during the propulsion cycle, which were correlated with the level of expertise: from arcing in novice to semi-circular in expert $[4,16]$. In 2014, Vegter et al. [29] investigated the effect of a low-intensity uninstructed practice in naive ablebodied subjects and showed an increase in gross mechanical efficiency accompanied with a decrease of cycle frequency and negative work per push. However, in contrast with the results of Hwang et al. [30], Vegter et al. [29] found an increase in propelling torque within the training program, resulting in higher shoulder loads [31].

Wheelchair start-up is a highly constraining task for the upper extremity. Indeed, during start-up, shoulder loads were found to be between 1.8 and 3.5 times those observed during steady-state propulsion [12,32]. In addition, start-up occurs from 200 to 350 times in a day [33,34]. As a consequence, it may be possible that during this task, subjects do not tend to exclusively decrease mechanical work but also tend to preserve their musculoskeletal integrity by limiting shoulder loads. In this case, the motor learning paradigm would be different between propulsion and startup. Indeed, some preliminary results have suggested that, over a certain level of shoulder loading, mechanical power decreased, whereas the mechanical work of the task still increased [35].

The purpose of this study was to investigate the evolution of shoulder kinetics during start-up and propulsion with a hand-rim wheelchair within the initial phase of a low-intensity uninstructed training. We hypothesized that net joint moments at the shoulder during start-up would quickly decrease in the course of the learning process. On the opposite, based on previous results [28,29,31], an increase of shoulder net joint moment was expected for the propulsion task.

\section{Materials and methods}

\section{Subjects}

Following approval by the relevant ethics committee (CPP Paris VI Pitié Salpétrière, France no. 2014-A01203-44), 17 able-bodied volunteers (4 females; 13 males) participated to this study. Subjects were 25.0 years old (SD: 3.4 ; range: $23-37$ y.o.); height: $1.78 \mathrm{~m}$ (SD: $0.07 \mathrm{~m}$, range: $1.63-1.97 \mathrm{~m}$ ); weight: $71.6 \mathrm{~kg}$ (SD: $9.1 \mathrm{~kg}$; range: $60-98 \mathrm{~kg}$ ) and body mass index: $22.6 \mathrm{~kg} / \mathrm{m}^{2}$ (SD: $1.6 \mathrm{~kg} / \mathrm{m}^{2}$, range: $20.0-25.4 \mathrm{~kg} / \mathrm{m}^{2}$ ). Able-bodied subjects were recruited because they provided a more homogeneous sample [28,29,31] and because their adaptations would not be influenced by specific muscle weaknesses or lack of control.

Participants had no prior experience with MWC locomotion and none of them presented any upper limb pain or injury before and during the completion of the study. They were informed of the protocol and signed a written informed consent form prior to their participation, allowing both collection and use of their anonymous data reported in this manuscript.

\section{Protocol}

The design of the training program was largely inspired from the study of Vegter et al. [29] and consisted in about $120 \mathrm{~min}$ of uninstructed practice distributed over 4 weeks. The first and last $15 \mathrm{~min}$ of the training program were dedicated to measurement sessions.

The measurement sessions consisted in three blocks of about 5 min during which the participant had to perform a succession of tasks (including start-up, propulsion, slalom and U-turn with various turning radii) successively announced by an assistant. The volunteer was asked to perform these tasks at a self-selected comfortable pace. Two minutes of rest were awarded between blocks. No information was given to the participant on the way to perform these tasks. All the subjects used the same instrumented wheelchair during the measurement sessions.

Between measurement sessions, the participant was asked to perform twice a week, $16 \mathrm{~min}$ of practice split into $8 \mathrm{~min}$ blocks with at least $1.5 \mathrm{~h}$ between them. During these training sessions, subjects had to move freely on a hard smooth level ground, performing start-ups, propulsions, turns, slaloms and reverse propulsions. Training was performed using four hand-rim wheelchairs (Otto Bock Voyager; Invacare Kuschall KSL; Vermeiren D200 and Vermeiren Eclips $30^{\circ}$ ) that differed in terms of geometry, wheel type, mass and inertia. The subject was asked to change of wheelchair every $4 \mathrm{~min}$ in order to limit accommodation to the settings of a single wheelchair and to favour the exploration of joint kinematics and kinetics through wheelchair characteristics. The subject received no information on how to improve his locomotion during the entire training program.

Finally, this training protocol was a low dose and low intensity uninstructed training program that should have prevented muscular and physiological adaptations. In other words, changes in biomechanical variables would be a motor learning only reflecting neurological adaptations.

\section{Measurement procedure}

Measurement procedure included the collection of both kinematic and kinetic data. Reflective markers were placed on both the subject and the wheelchair and their 3D locations were recorded at $100 \mathrm{~Hz}$ by 13 cameras of an optoelectronic motion capture system (Vicon system, hardware: T10 and T20S cameras; Nexus software; Oxford Metrics, Oxford, UK). Markers were placed on the spinous processes of the seventh cervical and eighth thoracic vertebrae, the manubrium, and the xiphoïd process for the torso; on the superior part of the acromion and on the medial and lateral epicondyles of the humerus for the arm; on the ulnar and radial styloïd processes for the forearm; and on the second and fifth metacarpal heads for the hand. Additionally, a technical platemounted markers cluster was attached to the arm and another technical marker was fixed on the distal third of the radius to assist in motion capture data collection. Wheelchair kinematics was recorded through four reflective markers placed on the chassis and on both rear-wheels centres.

Kinetic data were obtained through a wireless instrumented wheelchair allowing both forces and torques applied by the hand on the hand-rim to be recorded at a $100 \mathrm{~Hz}$ frequency (TSR-mesures, France $[36,37])$. Signals were corrected from baseline values according to already detailed procedures $[38,39]$. 
Synchronization between systems was ensured by knocking the instrumented hand-rim with a rubber hammer equipped with a reflective marker at the beginning of each block of locomotion. The shock created a synchronization event noticeable on both the hand-rim force signal and the hammer marker velocity.

\section{Data processing}

Start-up and propulsion cycles were identified in every block of recorded locomotion to provide kinematic and kinetic data for individual tasks.

Segment coordinate systems of the thorax, arm, forearm and hand were defined following the standards proposed by the International Society of Biomechanics [40]. Joint kinematics was obtained through a three-dimensional linked-segment model, using an inverse kinematics algorithm implemented in OpenSim [41]. The model used included hand, forearm bones, humerus, clavicle, scapula and thorax. It was derived from Saul et al. [42], with the addition of 2 degrees of freedom (dof) between the clavicle and the thorax. These additional dof allowed to take into account the forward-backward and upward-downward motions of the glenohumeral joint centre with respect to the thorax reference frame.

Body segment inertial parameters were scaled from subjects' anthropometry preserving mass distribution of the generic model [42]. Then, shoulder net moments were obtained using an inverse dynamics process and were finally expressed in the thorax orthonormal coordinate system, centred on the humeral head centre, to allow clinical interpretation [7,34]. Definitions of parameters reported in the present paper are given in Table 1.

\section{Data analysis}

For each subject, all kinematic and kinetic data were split into single tasks, and time-normalized with respect to the task duration. Biomechanical variables (Table 1) were averaged over the different cycles (at least six per block) performed by each individual. Results were then averaged among the whole population.

\section{Results}

All participants followed the entire uninstructed learning protocol and performed the two measurement sessions. For clarity purposes, the results are presented for the initial (T1) and final (T2) blocks of the pre-learning test and for the final block (T3) of the post-learning session. In this way, the evolution between T1 and T2 represented the very short-term adaptation during the first 15 min, whereas the comparison with T3 gives the adaptation during the 3 weeks uninstructed training program.

\section{Spatiotemporal parameters}

Comparison of temporal parameters (cycle, push and recovery times) before and after the 3 weeks of uninstructed training program did not show major changes for both propulsion and startup tasks (Table 2). Indeed, for propulsion, cycle time remained constant between T1 and T3 and only decreased from $1.90 \mathrm{~s}$ at T1 to $1.80 \mathrm{~s}$ at T3 for start-up. However, in spite of this relative consistency in task duration, a slight increase of the recovery time accompanied by a shortening of the push time was observed in both propulsion and start-up tasks (Table 2).

Concerning MWC velocity, an increase in self-selected comfort velocity was observed, both during propulsion $(\mathrm{T} 1: 0.70 \mathrm{~m} / \mathrm{s}$; T2: $0.86 \mathrm{~m} / \mathrm{s}$; T3: $1.02 \mathrm{~m} / \mathrm{s}$ ) and start-up (T1: $0.55 \mathrm{~m} / \mathrm{s}$; T2: $0.69 \mathrm{~m} / \mathrm{s}$; T3: $0.81 \mathrm{~m} / \mathrm{s}$ ).

\section{Shoulder loading during the push phase}

During the push phase, flexion (around the medio-lateral axis of the thorax) and internal rotation (around the longitudinal axis of the thorax) moments were the major components of the global shoulder net joint moment for both start-up and propulsion tasks. Peak values of these moments increased with learning for both propulsion and start-up tasks (Table 3). Peak value of the global net joint moment also increased with learning: from $15.4 \mathrm{Nm}$ at T1 to $22.6 \mathrm{Nm}$ at T3 for propulsion; and from $22.0 \mathrm{Nm}$ at T1 to $30.8 \mathrm{Nm}$ at T3 for start-up cycles. For both start-up and propulsion tasks, flexion angular impulse increased for short-term learning (between $\mathrm{T} 1$ and $\mathrm{T} 2$ ) but remained almost constant between T2

Table 2. Temporal parameters of MWC locomotion cycles.

\begin{tabular}{lccccc}
\hline & \multicolumn{2}{c}{ Propulsion } & & \multicolumn{2}{c}{ Start-up } \\
\cline { 2 - 3 } \cline { 5 - 6 } & T1 & T3 & & T1 & T3 \\
\hline Push time (s) & $0.65(0.10)$ & $0.60(0.05)$ & & $1.45(0.23)$ & $1.30(0.15)$ \\
Recovery time $(\mathrm{s})$ & $0.55(0.13)$ & $0.60(0.18)$ & & $0.44(0.12)$ & $0.50(0.19)$ \\
Task time (s) & $1.20(0.20)$ & $1.20(0.21)$ & & $1.90(0.31)$ & $1.80(0.28)$ \\
Velocity (m/s) & $0.70(0.12)$ & $1.02(0.13)$ & & $0.55(0.09)$ & $0.81(0.10)$ \\
\hline
\end{tabular}

T1: mean of initial pre-training trials; T3: mean of post-training trials. Standard deviations are presented between brackets.

Table 1. Biomechanical variables.

\begin{tabular}{|c|c|c|}
\hline Parameter & Unit & Description \\
\hline Push time & s & Duration of the push phase. Defined by the contact of the hands on the hand-rims \\
\hline Recovery time & $\mathrm{s}$ & $\begin{array}{l}\text { Duration of the free-wheeling phase. Defined by the absence of contact with the } \\
\text { hand-rims }\end{array}$ \\
\hline Task time & $\mathrm{S}$ & Total duration of the push and recovery phases \\
\hline Velocity & $\mathrm{m} / \mathrm{s}$ & $\begin{array}{l}\text { Velocity of the midpoint between rear wheel centre (mean value for propulsion cycles, } \\
\text { final value for start-up cycles) }\end{array}$ \\
\hline Abduction/adduction shoulder moment $M_{\mathrm{X}}$ & $\mathrm{Nm}$ & $\begin{array}{l}\text { Net shoulder moment following the fore-aft axis of the thorax. Abduction moments are } \\
\text { positive values }\end{array}$ \\
\hline Internal/external rotation shoulder moment $M_{Y}$ & $\mathrm{Nm}$ & $\begin{array}{l}\text { Net shoulder moment following the upward-downward axis of the thorax. Internal } \\
\text { rotation moments are positive values }\end{array}$ \\
\hline Flexion/extension shoulder moment $M_{Z}$ & $\mathrm{Nm}$ & $\begin{array}{l}\text { Net shoulder moment following the medio-lateral axis of the thorax. Flexion moments } \\
\text { are positive values }\end{array}$ \\
\hline Global shoulder moment $M_{\mathrm{G}}$ & $\mathrm{Nm}$ & Vectorial sum of $M_{\mathrm{x}}, M_{\mathrm{x}}$ and $M_{\mathrm{z}}$ \\
\hline Shoulder push flexion impulse $I^{+}\left(M_{\mathrm{Z}}\right)$ & $\mathrm{Nm} \mathrm{s}$ & Time integration of the shoulder flexion moment during the push phase \\
\hline Shoulder recovery extension impulse $I^{-}\left(M_{Z}\right)$ & $\mathrm{Nm} \mathrm{s}$ & Time integration of the shoulder extension moment during the recovery phase \\
\hline Shoulder positive work $W^{+}$ & J & Positive mechanical work of the shoulder moments \\
\hline Shoulder negative work $W^{-}$ & $\mathrm{J}$ & Negative mechanical work of the shoulder moments \\
\hline
\end{tabular}


Table 3. Shoulder kinetic parameters during the push phase.

\begin{tabular}{|c|c|c|c|c|c|c|}
\hline & \multicolumn{3}{|c|}{ Propulsion } & \multicolumn{3}{|c|}{ Start-up } \\
\hline & $\mathrm{T} 1$ & $\mathrm{~T} 2$ & $\mathrm{~T} 3$ & $\mathrm{~T} 1$ & $\mathrm{~T} 2$ & T3 \\
\hline$M_{X}^{\max }(\mathrm{Nm})$ & $1.5(2.0)$ & $5.2(6.5)$ & $4.6(5.8)$ & $3.6(3.8)$ & $5.0(4.1)$ & $5.0(4.4)$ \\
\hline$M_{y}^{\max }(\mathrm{Nm})$ & $3.1(1.7)$ & $4.2(1.8)$ & $5.5(2.5)$ & $5.9(2.3)$ & $8.8(2.5)$ & $11.3(2.6)$ \\
\hline$M_{7}^{\max }(\mathrm{Nm})$ & $9.4(3.6)$ & $17.0(8.1)$ & $18.7(10.5)$ & $19.3(8.1)$ & $23.6(8.0)$ & $28.1(9.7)$ \\
\hline$M_{\mathrm{G}}^{\max }(\mathrm{Nm})$ & $15.4(3.4)$ & $20.9(9.2)$ & $22.6(10.1)$ & $22.0(7.6)$ & $26.3(8.2)$ & $38.0(9.5)$ \\
\hline$I^{+}\left(M_{\mathrm{z}}\right)(\mathrm{Nm} \mathrm{s})$ & $2.2(1.2)$ & $4.6(2.9)$ & $4.5(2.7)$ & $12.5(6.8)$ & $15.3(6.3)$ & $15.9(6.0)$ \\
\hline$W^{-}(J)$ & $2.4(1.6)$ & $2.1(1.2)$ & $1.9(0.9)$ & $1.9(1.7)$ & $1.6(1.3)$ & $1.6(1.3)$ \\
\hline$W^{+}(J)$ & $2.4(1.0)$ & $6.2(5.1)$ & $6.9(4.6)$ & $5.6(2.5)$ & $9.1(4.5)$ & $12.2(5.2)$ \\
\hline
\end{tabular}

T1: mean of initial pre-training trials; T2: mean of final pre-training trials; T3: mean of post-training trials. Standard deviations are presented between brackets.

Table 4. Shoulder kinetic parameters during the recovery phase.

\begin{tabular}{|c|c|c|c|c|c|c|}
\hline & \multicolumn{3}{|c|}{ Propulsion } & \multicolumn{3}{|c|}{ Start-up } \\
\hline & $\mathrm{T} 1$ & $\mathrm{~T} 2$ & T3 & T1 & $\mathrm{T} 2$ & T3 \\
\hline$\overline{M_{X}^{\min }(\mathrm{Nm})}$ & $-5.4(2.5)$ & $-5.9(1.9)$ & $-5.5(2.3)$ & $-5.5(3.3)$ & $-6.3(2.2)$ & $-5.3(2.4)$ \\
\hline$M_{Y}^{\hat{m i n}}(\mathrm{Nm})$ & $-1.5(1.0)$ & $-2.0(1.0)$ & $-3.2(1.8)$ & $-1.2(0.8)$ & $-1.7(1.0)$ & $-2.2(0.9)$ \\
\hline$M_{Z}^{\min }(\mathrm{Nm})$ & $-12.3(3.6)$ & $-13.5(4.0)$ & $-12.0(3.9)$ & $-11.6(3.8)$ & $-12.6(3.5)$ & $-10.5(3.6)$ \\
\hline$M_{\mathrm{G}}^{\max }(\mathrm{Nm})$ & $13.5(3.7)$ & $14.6(4.0)$ & $13.4(4.2)$ & $12.9(4.3)$ & $14.1(3.4)$ & 11.9 (3.7) \\
\hline $\mathrm{I}^{-}\left(M_{\mathrm{Z}}\right)(\mathrm{Nm} \mathrm{s})$ & $-5.1(2.0)$ & $-6.4(2.5)$ & $-4.5(2.0)$ & $-4.1(1.9)$ & $-5.5(2.4)$ & $-3.7(2.2)$ \\
\hline$W^{-}(J)$ & $-0.2(0.2)$ & $-0.6(0.5)$ & $-0.2(0.2)$ & $-0.1(0.1)$ & $-0.3(0.2)$ & $-0.2(0.3)$ \\
\hline$W^{+}(\mathrm{J})$ & $6.3(2.3)$ & $7.6(2.4)$ & $7.7(2.6)$ & $5.8(2.6)$ & $7.4(2.5)$ & $7.2(3.0)$ \\
\hline
\end{tabular}

T1: mean of initial pre-training trials; T2: mean of final pre-training trials; T3: mean of post-training trials. Standard deviations are presented between brackets.

and T3 (Table 3). Finally, shoulder positive work during each push phase increased within the uninstructed training program for both propulsion (T1: $2.4 \mathrm{~J} ; \mathrm{T} 2: 6.2 \mathrm{~J}$; T3: $6.9 \mathrm{~J}$ ) and start-up (T1: $5.6 \mathrm{~J} ; \mathrm{T} 2$ : $9.1 \mathrm{~J} ;$ T3: $12.2 \mathrm{~J})$. A slight decrease of the shoulder negative work was observed, for both start-up and propulsion tasks.

\section{Shoulder loading during the recovery phase}

During the recovery phase, extension (around the medio-lateral axis of the thorax) and abduction (around the fore-aft axis of the thorax) moments were the major components of the shoulder net joint moments for both start-up and propulsion tasks. Peak moments and angular impulses followed the same trend for both tasks: after a slight increase within the first $15 \mathrm{~min}$ of practice (T1 vs T2), shoulder loads finally decreased at T3, below the initial values obtained in T1 (Table 4). Still, there was an overall increase in shoulder positive work during the recovery phase, even if it remained at the same level between $\mathrm{T} 2$ and $\mathrm{T} 3$.

\section{Individual differences in learning strategies}

Two main adaptation strategies, illustrated in Figure 1, were observed for the push phase during the learning process. Eleven subjects, classified as "increasers" (INC group), reached higher net shoulder moments at T3 than at T2, for both start-up and propulsion tasks. Individuals within the INC group increased the peak shoulder moment gradually for start-up (T2: $+34 \%$ with respect to $\mathrm{T} 1$; $\mathrm{T} 3:+81 \%$ with respect to $\mathrm{T} 1)$ and for propulsion (T2: $+46 \%$; T3: $+73 \%)$. The main contribution to this increase in shoulder moment was the flexion moment, with a shoulder push flexion impulse ranging from $10.7 \mathrm{Nm} \mathrm{s}$ (start-up, T1) to $19.3 \mathrm{Nm}$ s (startup, T3). It resulted in an increase of shoulder positive work with learning among this group (Table 5).

The six other participants (DEC group) managed to limit their shoulder loading during the push phase, especially the flexion/ extension moment, while succeeding in increasing the MWC velocity. In average, the shoulder net moments within the DEC group were slightly modified between $\mathrm{T} 1$ and $\mathrm{T} 2$ for both propulsion (from 14.0 to $16.3 \mathrm{Nm}$ ) and start-up (from 25.9 to $25.8 \mathrm{Nm}$ ) and decreased at T3. The flexion moment largely contributed to this trend. For instance, the shoulder push impulse during start-up dropped to $9.7 \mathrm{Nm}$ s at T3, with an initial value of $16.0 \mathrm{Nm}$ s at T1. As shown in Table 5, shoulder positive work increased with a lower rate in the DEC group than in the INC group.

The recovery phase displayed a homogeneous trend for the whole population, since each subject reduced, or at least maintained, shoulder moments after the training period. It was not the case for the shoulder positive work during the recovery phase, which was higher in T3 than in T1 for 12 subjects, from both groups.

If average anthropometric characteristics showed that DEC individuals tended to be smaller and lighter (height: $1.76 \mathrm{~m}, \mathrm{SD}$ : $0.04 \mathrm{~m}$; weight: $66.8 \mathrm{~kg}$, SD: $5.60 \mathrm{~kg}$ ) than individuals of the INC group (height: $1.78 \mathrm{~m}, \mathrm{SD}: 0.08 \mathrm{~m}$; weight: $72.9 \mathrm{~kg}, \mathrm{SD}: 8.10 \mathrm{~kg}$ ), a significant overlap between the two groups was also observed.

\section{Discussion}

\section{Spatiotemporal parameters}

During this study, the task duration was not spontaneously modified by the users during the initial phase of the learning process. Propulsion cycles duration was in average higher than the values reported in the literature $[22,28,29,31]$, which may be explained by the use of a field wheelchair in the present study, compared to studies performed on treadmills. For start-up, the duration of push phases was consistent with the study of Kloosterman et al. [34], who reported a push time of $1.3 \mathrm{~s}$ for experienced wheelchair users. However, during the very first minutes of practice (i.e., between T1 and T2), a slight increase of cycle time was observed, which was consistent with previous results on short-term learning $[28,29,31]$. This highlighted the fact that the propulsion technique is not modified during the learning process following a continuous trend. Finally, while maintaining a similar total task time at T3, 

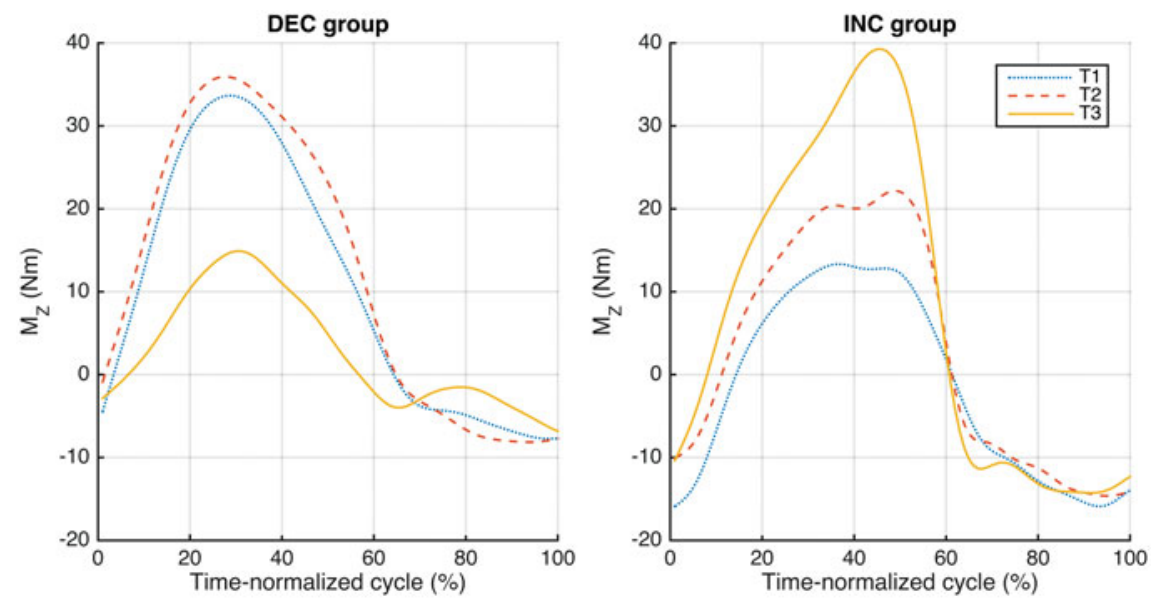

Figure 1. Typical evolutions of flexion/extension moment patterns during the learning process, for the DEC (left) and INC (right) groups. Each graph represents the results for one individual from the population.

Table 5. INC/DEC kinetic parameters during the push phase.

\begin{tabular}{|c|c|c|c|c|c|c|}
\hline & \multicolumn{3}{|c|}{ INC group } & \multicolumn{3}{|c|}{ DEC group } \\
\hline & $\mathrm{T} 1$ & $\mathrm{~T} 2$ & T3 & $\mathrm{T} 1$ & $\mathrm{~T} 2$ & T3 \\
\hline Start-up: $M_{\mathrm{G}}^{\max }(\mathrm{Nm})$ & $19.9(7.0)$ & $26.6(8.6)$ & $36.1(6.6)$ & $25.9(7.6)$ & $25.8(8.2)$ & $21.1(4.9)$ \\
\hline Propulsion: $M_{\mathrm{G}}^{\max }(\mathrm{Nm})$ & $16.2(3.6)$ & $23.7(10.2)$ & $28.0(8.3)$ & $14.0(2.7)$ & $16.3(5.0)$ & $12.8(3.6)$ \\
\hline Start-up: $I^{+}\left(M_{\mathrm{Z}}\right)(\mathrm{Nm} \mathrm{s})$ & $10.7(6.6)$ & $15.4(6.8)$ & $19.3(3.6)$ & $16.0(6.0)$ & $15.1(5.9)$ & $9.7(4.1)$ \\
\hline Propulsion: $\mathrm{I}^{+}\left(\mathrm{M}_{\mathrm{Z}}\right)(\mathrm{Nm} \mathrm{s})$ & $2.2(0.7)$ & $5.0(3.2)$ & $5.7(2.2)$ & $2.4(1.8)$ & $4.0(2.4)$ & $1.1(1.7)$ \\
\hline Start-up: $W^{+}(J)$ & $5.0(2.4)$ & $9.2(5.0)$ & $14.6(4.7)$ & $6.6(2.6)$ & $8.8(4.1)$ & $7.7(2.1)$ \\
\hline Propulsion: $W^{+}(\mathrm{J})$ & $2.5(1.0)$ & $7.5(6.0)$ & $9.1(4.3)$ & $2.2(1.1)$ & $4.1(1.8)$ & $2.9(1.1)$ \\
\hline
\end{tabular}

T1: mean of initial pre-training trials; T2: mean of final pre-training trials; T3: mean of post-training trials. Standard deviations are presented between brackets.

subjects spontaneously reduced the push time to increase the duration of the recovery phase. This natural evolution confirmed the acquisition of confidence and skills on the wheelchair with practice, revealing a more efficient handling of the MWC behaviour.

In contrast with cycle time, the self-selected comfort wheelchair velocity adopted by subjects increased with learning, for both short-term (T1 vs T2) and mid-term learning (T1 vs T3). This evolution was already mentioned during the very first minutes of practice in naive able-bodied subjects [27]. However, many other studies investigating the effect of uninstructed MWC practice on propulsion technique were performed on a treadmill $[28,29,31]$ thus imposing a constant speed before and after the learning protocol. Given the conclusions of the present study, such protocols might have induced a bias in describing the natural evolution of over-ground MWC propulsion technique even if these studies remain interesting to describe the evolution at a controlled external mechanical power. In addition, if the treadmill velocity (1.11 $\mathrm{m} / \mathrm{s}$ ) chosen in previous studies $[28,29,31]$ could be suitable to experienced MWC users, our results (from $0.70 \mathrm{~m} / \mathrm{s}$ at T1 to 1.02 $\mathrm{m} / \mathrm{s}$ at T3) suggest that lower velocities should be preferred for naive young able-bodied subjects.

\section{Shoulder loading during the push phase}

At each step of the protocol, the push phase involved mainly flexion and internal rotation moments, for both start-up and propulsion, which confirms results of the literature for steady-state propulsion [7], with the same coordinate system. Our results also indicate that shoulder net moments were in average 1.4 times higher during start-up than during propulsion, which is slightly lower than the 1.6 to 3.1 ratio described by Koontz et al. [12] for hand-rim kinetics.

The increase of shoulder net moments and angular impulses within the first $15 \mathrm{~min}$ of practice, reported in the literature $[28,29,31]$ for propulsion on a treadmill, was also observed in the present study. This increase not only occurred for propulsion and during the first minutes of practice, but also for start-up and throughout the 3 weeks of the uninstructed training period. Because the push time was not notably altered, it is mainly the increase of shoulder moments and work per push that enabled subjects to increase the linear speed of the wheelchair. Our hypothesis is thus confirmed for the propulsion task, but contrary to our expectations, start-up displayed a similar evolution with learning. This trend could be explained by the efforts developed by the user that may remain below the critical level of pain. This could lead to a learning process mainly driven by the building of self-confidence on the wheelchair rather than by a strategy favouring upper limbs preservation.

\section{Shoulder loading during the recovery phase}

Conversely to the push phase, the recovery phase has not often been investigated in previous biomechanical analyses of MWC locomotion, because most of these studies were focused on handrim kinetics $[22,25,26,28,29]$ and there is no contact between the hands and the hand-rims during the recovery phase.

During the very first minutes of practice, the amplitude of net joint moments increased for the recovery phase. However, after the whole training protocol, subjects limited the articular loading in the backwards motion of their arms. The increase in shoulder positive work occurring in the meantime implied that the 
kinematics of the arm was the main factor influencing the motor learning in the recovery phase. After an initial session during which subjects made jerky movements for the transition between push and recovery phases, subjects chose a more continuous and economical motion between the end of the push and the next grasp of the hand-rim. It is consistent with the findings of other studies about the links between hand patterns and the level of expertise on the wheelchair $[13,16]$.

\section{Individual differences in learning strategies}

The emerging of INC and DEC trends was only noticeable after the 3-week period of practice. Indeed, for most subjects of the DEC group, the decrease in the shoulder loading did not occur at T2, but became obvious at T3. It can be explained by the fact that during the first $15 \mathrm{~min}$ of practice, subjects initially explored the range of motion before defining their personal propulsion strategy. That is why the learning process cannot be seen as continuous, especially between $\mathrm{T} 1$ and $\mathrm{T} 2$, but also between $\mathrm{T} 1$ and $\mathrm{T} 3$. However, it suggests that long-term learning strategies observed in studies about the very first minutes of MWC practice [29] may differ when considering a longer learning timescale.

The learning paradigm that was hypothesized in this study was not confirmed, because individuals belonging to DEC or INC group were the same for start-up and propulsion, except for two subjects. Conversely, this study led to another conclusion considering our hypothesis: the motor learning process is not taskdependent (for start-up and propulsion) but subject-dependent. This implied that some subjects (INC group) imposed higher stress on their shoulder as they gain experience, while others (DEC group) used practice as a way to preserve their musculoskeletal integrity, regardless of the performed task.

That is why future research should deeper investigate the factors leading individuals to choose the INC or DEC learning strategy, especially by analysing muscle forces on the shoulder complex, for instance.

\section{Limitations}

There were some limitations in this study. First of all, because the protocol involved young able-bodied adults, application of the results to other populations should be made cautiously. Persons using a MWC temporarily after lower limb surgery, lower limb amputees or spinal cord injured patients with a lesion under the 12th thoracic vertebra can, however, be seen as populations for which the applications of the results are more straightforward. Conducting similar studies among a larger and more diverse population may help in broadening the learning spectrum.

Due to diverse practical reasons, only the right-side of the body was used for inverse dynamics. It might be interesting to investigate the possible impact of learning on the symmetry of upper limbs kinematics and kinetics. Because net joint moments are only global estimates of what is occurring at the joint level, future research should focus on muscular activations, in order to assess glenohumeral joint contact forces.

\section{Conclusion}

The results of this study show that biomechanical modifications of the propulsion technique occurred from the early stages of autonomous MWC practice. The push phase was critical, since the learning process enabled subjects to increase the wheelchair velocity, at the cost of higher shoulder moments and mechanical work. Contrary to our expectations, the evolution was similar for start-up and propulsion tasks. Conversely, skills acquisition enabled users to limit the shoulder loading during the recovery phase, through a better synchronization of the backward motion of the upper limb. Finally, two distinct trends, corresponding to different motor learning strategies, emerged: whereas some subjects increased shoulder net moments to reach a higher MWC velocity, others managed to limit shoulder loading. The motor learning paradigm seemed to be changing according to individuals, rather than to the task. These findings underline the need of individualizing rehabilitation programs, to help MWC users find their personal optimal propulsion technique. Future long-term longitudinal studies may also be conducted to evaluate the possible link between shoulder loading strategies and musculoskeletal disorders occurrence.

\section{Disclosure statement}

The authors report no declaration of interest.

\section{ORCID}

Samuel Hybois (D) http://orcid.org/0000-0003-3838-2860

Joseph Bascou (iD http://orcid.org/0000-0001-5290-8158

Hélène Pillet (iD http://orcid.org/0000-0002-0263-5910

Christophe Sauret (D) http://orcid.org/0000-0002-6196-2698

\section{References}

[1] Ville I, Ravaud J-F, Mahut D. Les utilisateurs du fauteuil roulant en France. Le fauteuil roulant manuel: choix et réglages [Manual wheelchair users in France, in the manual wheelchair: choice and settings]. 2011. p. 59-68.

[2] Improving services for wheelchair users and carers: Good Practice Guide - learning from the Wheelchair Services Collaborative. London: Department of Health and the NHS Modernisation Agency; 2005.

[3] Ackermann M, Leonardi F, Costa HR, et al. Modeling and optimal control formulation for manual wheelchair locomotion: the influence of mass and slope on performance. In: Proceedings of the 5th IEEE RAS/EMBS International Conference on Biomedical Robotics and Biomechatronics. IEEE; 2014. p. 1079-1084.

[4] Boninger ML, Dicianno BE, Cooper RA, et al. Shoulder magnetic resonance imaging abnormalities, wheelchair propulsion, and gender. Arch Phys Med Rehabil. 2003; 84:1615-1620.

[5] Nichols PJR, Norman PA, Ennis JR. Wheelchair user's shoulder? Shoulder pain in patients with spinal cord lesions. Scand J Rehabil Med. 1979;11:29-32.

[6] Finley MA, Rodgers MM. Prevalence and identification of shoulder pathology in athletic and nonathletic wheelchair users with shoulder pain: a pilot study. J Rehabil Res Dev. 2004;41:395-402.

[7] Mercer JL, Boninger M, Koontz A, et al. Shoulder joint kinetics and pathology in manual wheelchair users. Clin Biomech (Bristol, Avon). 2006;21:781-789.

[8] Robertson RN, Boninger ML, Cooper RA, et al. Pushrim forces and joint kinetics during wheelchair propulsion. Arch Phys Med Rehabil. 1996;77:856-864.

[9] Kulig K, Rao SS, Mulroy SJ, et al. Shoulder joint kinetics during the push phase of wheelchair propulsion. Clin Orthop Relat Res. 1998;354:132-143. 
[10] Veeger HEJ, Rozendaal LA, van der Helm FCT. Load on the shoulder in low intensity wheelchair propulsion. Clin Biomech (Bristol, Avon). 2002;17:211-218.

[11] Van Drongelen S, Van der Woude LH, Janssen TW, et al. Mechanical load on the upper extremity during wheelchair activities. Arch Phys Med Rehabil. 2005;86:1214-1220.

[12] Koontz AM, Cooper RA, Boninger ML, et al. A kinetic analysis of manual wheelchair propulsion during start-up on select indoor and outdoor surfaces. JRRD. 2005;42:447-458.

[13] Boninger ML, Souza AL, Cooper RA, et al. Propulsion patterns and pushrim biomechanics in manual wheelchair propulsion. Arch Phys Med Rehabil. 2002;83:718-723.

[14] de Groot S, Veeger HEJ, Hollander AP, et al. Effect of Wheelchair Stroke Pattern on Mechanical Efficiency. Am J Phys Med Rehabil. 2004;83:640-649.

[15] Qi L, Wakeling J, Grange S, et al. Patterns of shoulder muscle coordination vary between wheelchair propulsion techniques. IEEE Trans Neural Syst Rehabil Eng. 2014; 22:559-566.

[16] Slowik JS, Requejo PS, Mulroy SJ, et al. The influence of wheelchair propulsion hand pattern on upper extremity muscle power and stress. J Biomech. 2016;49:1554-1561.

[17] Rodgers MM, Keyser RE, Rasch EK, et al. Influence of training on biomechanics of wheelchair propulsion. J Rehabil Res Dev. 2001;38:505-511.

[18] Bougenot M-P, Tordi N, Betik AC, et al. Effects of a wheelchair ergometer training programme on spinal cord-injured persons. Spinal Cord. 2003;41:451-456.

[19] de Groot S, de Bruin M, Noomen SP, et al. Mechanical efficiency and propulsion technique after 7 weeks of lowintensity wheelchair training. Clin Biomech (Bristol, Avon). 2008;23:434-441.

[20] Tordi N, Mourot L, Chapuis A, et al. Effects of a primary rehabilitation programme on arterial vascular adaptations in an individual with paraplegia. Ann Phys Rehabil Med. 2009;52:66-73.

[21] de Groot S, Veeger HEJ, Hollander AP, et al. Consequence of feedback-based learning of an effective hand rim wheelchair force production on mechanical efficiency. Clin Biomech (Bristol, Avon). 2002;17:219-226.

[22] Leving MT, Vegter RJK, Hartog J, et al. Effects of visual feedback-induced variability on motor learning of handrim wheelchair propulsion. PLoS ONE. 2015;10:e0127311.

[23] Rice I, Gagnon D, Gallagher J, et al. Hand rim wheelchair propulsion training using biomechanical real-time visual feedback based on motor learning theory principles. J Spinal Cord Med. 2010;33:33-42.

[24] Blouin M, Lalumiere $\mathrm{M}$, Gagnon $\mathrm{DH}$, et al. Characterization of the immediate effect of a training session on a manual wheelchair simulator with haptic biofeedback: towards more effective propulsion. IEEE Trans Neural Syst Rehabil Eng. 2015;23:104-115.

[25] Van Der Woude LHV, Van Croonenborg JJ, Wolff I, et al. Physical work capacity after $7 \mathrm{wk}$ of wheelchair training: effect of intensity in able-bodied subjects. Med Sci Sports Exerc. 1999;31:331-341.

[26] de Groot S, Veeger HEJ, Hollander AP, et al. Short-term adaptations in co-ordination during the initial phase of learning manual wheelchair propulsion. J Electromyogr Kinesiol. 2003;13:217-228.
[27] Roux L, Hanneton S, Roby-Brami A. Shoulder movements during the initial phase of learning manual wheelchair propulsion in able-bodied subjects. Clin Biomech (Bristol, Avon). 2006;21(Suppl 1):S45-S51.

[28] Vegter RJK, Lamoth CJ, de Groot S, et al. Inter-individual differences in the initial 80 minutes of motor learning of handrim wheelchair propulsion. PLoS One. 2014;9:e89729.

[29] Vegter RJK, de Groot S, Lamoth CJ, et al. Initial skill acquisition of handrim wheelchair propulsion: a new perspective. IEEE Trans Neural Syst Rehabil Eng. 2014;22:104-113.

[30] Hwang S, Kim S, Kim Y. Torque and power outputs on skilled and unskilled users during manual wheelchair propulsion. Conf Proc IEEE Eng Med Biol Soc. 2012;2012: 4820-4822.

[31] Vegter RJK, Hartog J, de Groot S, et al. Early motor learning changes in upper-limb dynamics and shoulder complex loading during handrim wheelchair propulsion. J NeuroEngineering Rehabil. 2015;12:26.

[32] Westerhoff P, Graichen F, Bender A, et al. Measurement of shoulder joint loads during wheelchair propulsion measured in vivo. Clin Biomech (Bristol, Avon). 2011;26:982-989.

[33] Tolerico ML, Ding D, Cooper RA, et al. Assessing mobility characteristics and activity levels of manual wheelchair users. J Rehabil Res Dev. 2007;44:561.

[34] Kloosterman MGM, Buurke JH, Schaake L, et al. Clinical biomechanics exploration of shoulder load during hand-rim wheelchair start-up with and without power-assisted propulsion in experienced wheelchair users. Clin Biomech (Bristol, Avon). 2016;34:1-6.

[35] Sauret C, Vaslin P, Dumas R, et al. Computation of the mechanical power of a manual wheelchair user in actual conditions: preliminary results. Comput Methods Biomech Biomed Eng. 2012;15(Suppl 1):173-174.

[36] Dabonneville $M$, Vaslin $P$, Kauffmann $P$, et al. self-contained wireless wheelchair ergometer designed for biomechanical measures in real life conditions. Technol Disabil. 2005;17:63-76.

[37] Sauret C, Vaslin P, Lavaste F, et al. Effects of user's actions on rolling resistance and wheelchair stability during handrim wheelchair propulsion in the field. Med Eng Phys. 2013;35:289-297.

[38] Sauret C, Dabonneville $M$, Couétard $Y$, et al. Zeroing of sixcomponent handrim dynamometer for biomechanical studies of manual wheelchair locomotion. Comput Methods Biomech Biomed Eng. 2014;17:416-422.

[39] Sauret C, Couetard Y, Vaslin P. Dynamic calibration of a wheelchair six-component wheel dynamometer rolling on the floor. Comput Methods Biomech Biomed Eng. 2011;14(suppl 1):67-69.

[40] Wu G, van der Helm FCT, (DirkJan) Veeger HEJ, et al. ISB recommendation on definitions of joint coordinate systems of various joints for the reporting of human joint motionPart II: shoulder, elbow, wrist and hand. J Biomech. 2005;38:981-992.

[41] Delp SL, Anderson FC, Arnold AS, et al. OpenSim: opensource software to create and analyze dynamic simulations of movement. IEEE Trans Biomed Eng. 2007;54:1940-1950.

[42] Saul KR, Hu X, Goehler CM, et al. Benchmarking of dynamic simulation predictions in two software platforms using an upper limb musculoskeletal model. Comput Methods Biomech Biomed Eng. 2015;18:1445-1458. 